\title{
Evaluation of Salmon, Tuna, and Beef Freshness Using a Portable Spectrometer
}

\author{
Eui Jung Moon ${ }^{1}$, Youngsik Kim ${ }^{2}$, Yu Xu ${ }^{1}$, Yeul Na ${ }^{2}$, Amato J. Giaccia ${ }^{1}$ and Jae Hyung Lee ${ }^{2, *}$ \\ 1 Department of Radiation Oncology, Stanford University, Stanford, CA 94305, USA; \\ ejung12@stanford.edu (E.J.M.); yxu33@stanford.edu (Y.X.); giaccia@stanford.edu (A.J.G.) \\ 2 Stratio Inc., Palo Alto, CA 94303, USA; youngsik.kim@stratiotechnology.com (Y.K.); \\ yeul.na@stratiotechnology.com (Y.N.) \\ * Correspondence: jaehyung.lee@stratiotechnology.com; Tel.: +1-650-796-7456
}

Received: 22 June 2020; Accepted: 30 July 2020; Published: 1 August 2020

check for updates

\begin{abstract}
There has been strong demand for the development of an accurate but simple method to assess the freshness of food. In this study, we demonstrated a system to determine food freshness by analyzing the spectral response from a portable visible/near-infrared (VIS/NIR) spectrometer using the Convolutional Neural Network (CNN)-based machine learning algorithm. Spectral response data from salmon, tuna, and beef incubated at $25{ }^{\circ} \mathrm{C}$ were obtained every minute for $30 \mathrm{~h}$ and then categorized into three states of "fresh", "likely spoiled", and "spoiled" based on time and pH. Using the obtained spectral data, a CNN-based machine learning algorithm was built to evaluate the freshness of experimental objects. In addition, a CNN-based machine learning algorithm with a shift-invariant feature can minimize the effect of the variation caused using multiple devices in a real environment. The accuracy of the obtained machine learning model based on the spectral data in predicting the freshness was approximately $85 \%$ for salmon, $88 \%$ for tuna, and $92 \%$ for beef. Therefore, our study demonstrates the practicality of a portable spectrometer in food freshness assessment.
\end{abstract}

Keywords: food freshness; portable spectrometer; near-infrared; machine learning

\section{Introduction}

Freshness is one of the most important parameters to evaluate food quality, especially for raw food such as fish and meat. A majority of grocery stores and/or consumers rely on sensory methods such as appearance, odor, taste, or texture to assess food freshness. However, these methods are often both subjective and inaccurate, as they are heavily dependent on the skill level of the inspector.

Although qualitative measurement for freshness is more reliable and precise, it requires expensive, slow, and/or destructive methods [1,2]. Physicochemical methods measure water content, $\mathrm{pH}$ value, thiobarbituric acid reacted substance (TBARS), total volatile basic nitrogen (TVB-N), sugar, and nutrition, including vitamins. Although these methods are widely used in controlled environments such as large-scale food processing plants, they are either destructive or prone to cross-contamination. Microbiological methods provide useful information regarding bacterial spoilage, but accurate measurement takes time. Therefore, alternative methods are required for grocery stores and/or consumers, who are in need of prompt results for quality assessment. Most recently, a non-destructive and in situ analysis for a fish freshness monitoring system was proposed by a group of researchers using the optical $\mathrm{pH}$ sensor; however, the absolute $\mathrm{pH}$ values do not necessarily increase or decrease monotonically during the testing period, which make it challenging to assess the freshness without monitoring the whole period [3].

Freshness assessment using a visible/near-infrared (VIS/NIR) spectrometer has been proposed by previous studies [4,5]. However, this approach has several drawbacks, as most of the well-known 
spectrometers are both bulky and expensive. Moreover, due to unique spectral response features of each food, the data need to be analyzed in different ways depending on food types. More recently, a breakthrough in technology enabled the production of low-cost spectrometer on a "chip", leading to the development of consumer-scale NIR devices including LinkSquare [6-8].

In this study, we introduce a smart method using LinkSquare, which is a handheld spectrometer that was designed and built by ourselves and commercially available for sale to the public [7-12], to assess food freshness (Figure 1). Samples were scanned with a VIS/NIR portable spectrometer and spectral data was analyzed using the Convolutional Neural Network (CNN)-based machine learning algorithm to evaluate the freshness of the objects $[13,14]$. The conventional $\mathrm{pH}$ meter was used to categorize the freshness and to verify our results.

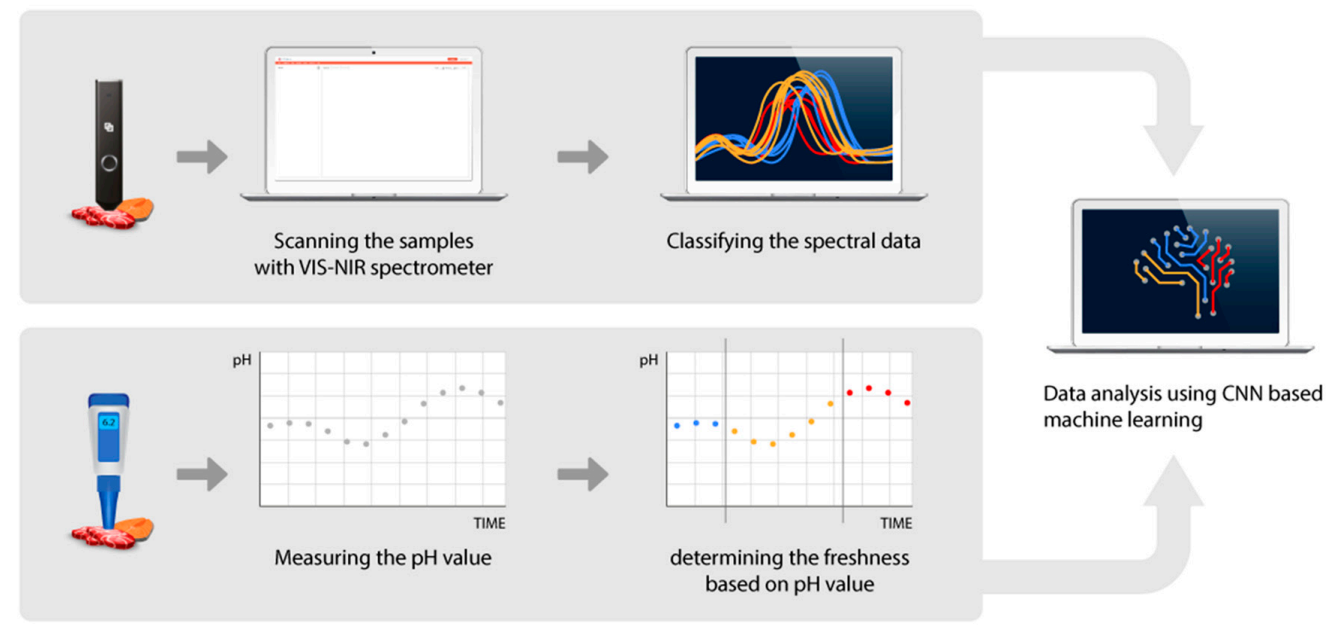

Figure 1. Overview of the food freshness estimation process using a portable visible/near-infrared (VIS-NIR) spectrometer, LinkSquare. As the rotting process of the sample continues, the spectral responses are collected over time with a LinkSquare device (above), and the $\mathrm{pH}$ values are measured at the same time (below) to determine the freshness of the samples. The spectral response data is used to train a machine learning model for the food freshness estimation (right).

\section{Materials and Methods}

\subsection{Instruments}

A PH60F pH meter (Apera Instruments, Columbus, OH, USA), which ranges from 2.00 to $16.00 \mathrm{pH}$ with an accuracy of $\pm 0.01 \mathrm{pH}$, was used for the $\mathrm{pH}$ measurement.

LinkSquare $^{\circledR}$ (www.linksquare.io), a portable VIS/NIR spectrometer, was used for the spectral response measurement. It was equipped with optical parts, an image sensor, a microcontroller unit (MCU), a white light-emitting diode (LED), a bulb, and a rechargeable Li-ion battery. The dimensions of the device were as small as $114.0 \times 23.9 \times 23.9 \mathrm{~mm}$, and its weight was $57 \mathrm{~g}$. The spectral range of the spectrometer was 400 to $1000 \mathrm{~nm}$ with a resolution of 5 to $30 \mathrm{~nm}$ (approximately $5 \mathrm{~nm} @ 500 \mathrm{~nm}, 10 \mathrm{~nm}$ @ 700 nm, and 20 nm @ 900 nm). Since each spectrometer unit was factory calibrated, data obtained from one unit can be applied to others with minimum error. These data will be called 'spectral response data' henceforth.

An AI Platform (ai.linksquare.io) to analyze the spectral response data using a CNN-based machine learning algorithm was developed by Stratio, Inc. (www.stratiotechnology.com).

\subsection{Sample Preparation and Experiment Setup}

The Atlantic salmon (Salmon salar) and Pacific salmon (Oncorhynchus nerka) were fresh and unfrozen, while tuna was frozen and imported from Indonesia. For beef, choice-grade USDA sirloin beef was used. 
Each sample was sliced into pieces with the size of $30 \times 30 \times 30 \mathrm{~mm}$ to be clamped together with a portable spectrometer by using a custom-built holder inside an incubator that maintains the temperature at $25^{\circ} \mathrm{C}$ to make a portable spectrometer direct contact to each food sample. Samples were placed on a glass container, and a spectrometer was installed on top of each sample, directly contacting the surface. A spectrometer emitted light toward the surface of a sample and measured the reflected light spectrum. Spectral response data were automatically recorded every minute and stored with its timestamp. More than 15 samples for each food and 9 devices were used in this study (Table 1). The spectra of 3 Atlantic salmon, 3 Pacific salmon, 8 tuna, and 8 beef samples were scanned for creating a training dataset, while the spectra of 12 Atlantic salmon, 12 Pacific salmon, 9 tuna, and 8 beef samples were scanned for a verification dataset.

To track the freshness, an additional 3 samples per sample for the spectrum measurement were prepared for the $\mathrm{pH}$ measurement. These extra samples underwent the same environment setup in the incubator. At the end of each period, which varied from one hour to five hours, a small (approximately $2 \mathrm{~g}$ ) piece was sliced out of each sample, and $\mathrm{pH}$ values were measured 20 times to minimize the measurement error. From these 20 measurements, the mean and the standard deviation were calculated.

Table 1. Multiple LinkSquare devices were used in this study, and we have labeled them from A to L (12 units). Each food samples were labeled as follows: Salmon Atlantic (SA), Salmon Pacific (SP), Tuna (T), and Beef (B). The number of 15,15, 17, and 16 samples were used respectively for both training and verification spectra data.

\begin{tabular}{|c|c|c|c|c|c|c|c|c|}
\hline \multirow{2}{*}{ Device } & \multicolumn{2}{|c|}{ Salmon (Atlantic) } & \multicolumn{2}{|c|}{ Salmon (Pacific) } & \multicolumn{2}{|c|}{ Tuna } & \multicolumn{2}{|c|}{ Beef } \\
\hline & Training & Verify & Training & Verify & Training & Verify & Training & Verify \\
\hline D1 & SA1 & SA4 & SP1 & SP4 & $\mathrm{T} 1$ & & B1 & \\
\hline D2 & SA2 & SA5 & SP2 & SP5 & $\mathrm{T} 2, \mathrm{~T} 3$ & & B2, B3 & \\
\hline D3 & SA3 & & SP3 & & $\mathrm{T} 4$ & & B4 & B9 \\
\hline D4 & & SA6, SA7 & & SP6, SP7 & T5 & T9 & B5 & B10 \\
\hline D5 & & SA8, SA9 & & SP8, SP9 & T6 & $\mathrm{T} 10, \mathrm{~T} 11$ & B6 & \\
\hline D6 & & $\begin{array}{l}\text { SA10, } \\
\text { SA11 }\end{array}$ & & $\begin{array}{l}\text { SP10, } \\
\text { SP11 }\end{array}$ & $\mathrm{T} 7$ & & B7 & \\
\hline D7 & & SA12 & & SP12 & $\mathrm{T} 8$ & & B8 & B11 \\
\hline D8 & & SA13 & & SP13 & & $\mathrm{T} 12$ & & B12, B13 \\
\hline D9 & & SA14 & & SP14 & & $\mathrm{T} 13, \mathrm{~T} 14$ & & B14 \\
\hline D10 & & SA15 & & SP15 & & T15 & & B15 \\
\hline D11 & & & & & & T16 & & B16 \\
\hline D12 & & & & & & T17 & & \\
\hline Total \# & 3 & 12 & 3 & 12 & 8 & 9 & 8 & 8 \\
\hline
\end{tabular}

\subsection{Staphylococcus Aureus Culture}

Staphylococcus aureus was isolated from food samples following protocols from the previous publication $[15,16]$. Meat and fish samples were cut into $5 \mathrm{~cm} \times 5 \mathrm{~cm}$ patches, which weigh approximately $50 \mathrm{~g}$, and incubated for $0,6-8$, and $24 \mathrm{~h}$. Samples were hand massaged with $10 \mathrm{~mL}$ of buffered peptone water (BPW, Simga-Aldrich) for $5 \mathrm{~min}$ and only fluid was collected using cheesecloth wipes (VWR). Then, $100 \mu \mathrm{L}$ of extracts were plated on Baird Parker (BP) selective media plates (Millipore Sigma) and incubated for $24 \mathrm{~h}$ at $37^{\circ} \mathrm{C}$. To count colonies, plate images were taken and analyzed using a customized python script (python 3.7, Wilimington, DE, USA) based on color thresholding and object size. The number of colonies was further validated by visual examination. 


\subsection{TBARS Assay}

First, $2 \mathrm{~g}$ of food samples were submerged in $5 \mathrm{~mL}$ of ice cold $10 \%$ trichloracetic acid (TCA) and incubated for $5 \mathrm{~min}$ on ice. Samples were homogenized in ice and spun down for $5 \mathrm{~min}$ at $4000 \mathrm{rpm}$. Supernatants were transferred and then spun down again for $5 \mathrm{~min}$ at 14,000 rpm. Supernatants were mixed with TBA reagents from a TBARS assay kit (BioAssay System) and incubated for $60 \mathrm{~min}$ at $100^{\circ} \mathrm{C}$. Optical density (OD) values were measured at $535 \mathrm{~nm}$ using a plate reader.

\subsection{CNN-Based Machine Learning Algorithm}

A CNN structure with 6 sets of convolution layers, rectified linear unit (ReLU) layers, and pooling layers followed by three fully connected layers was used in this study. Since the input was 1-Dimensional (D) instead of 2-D in the typical computer vision problem, a small modification was made on the convolution layers so that they perform only 1-D convolution. Except for the small modification, the neural network architecture used in this study is similar with that of AlexNet ${ }^{16}$. AlexNet with a shift-invariant feature can minimize the effect of the variation caused using multiple devices in a real environment. A machine learning model for each tested food category was made, which gives four models in total for this study.

The spectral response data that cover the full spectral range $(400-1000 \mathrm{~nm})$ were provided as input vectors of the CNN structure. Two light sources, an LED and a bulb, were used in LinkSquare ${ }^{\circledR}$, which is why one set of inputs consisted of two 1-D spectral response vectors $(600 \times 1)$. They were treated separately through the modified CNN structure, which consists of 1-D convolution layers and 1-D max pooling layers until the last pooling layer, and these were combined together to form a 1-D input vector of the first fully connected layer. Three categories ("fresh", "likely spoiled", and "spoiled") for each food type were properly encoded and were provided as output vectors of the last fully connected layer.

The training of the CNN-based machine learning algorithm was implemented using the Torch framework on a NVidia GeForce GTX Titan X. For each experimental setup, several runs of experiments were performed independently. Some of the runs became the training dataset, and the rest of them became a completely separated dataset for testing, which was also known as the verification dataset. The training dataset was divided into two smaller sets: $90 \%$ of them were used for actual training, while $10 \%$ of them were only for checking the current performance of the model by evaluating the accuracy. The division was done randomly among the scans from various samples and devices. The accuracy was calculated only using the aforementioned verification dataset.

\subsection{Sensitivity and Specificity}

The values of sensitivity and specificity are determined using the following equations:

$$
\begin{aligned}
& \text { Sensitivity }=\frac{\mathrm{TP}}{\mathrm{TP}+\mathrm{FN}} \\
& \text { Specificity }=\frac{\mathrm{TN}}{\mathrm{FP}+\mathrm{TN}}
\end{aligned}
$$

The concepts of True Positive (TP), True Negative (TN), False Positive (FP), and False Negative (FN) in the above equations can be easily defined with a binary classification test.

\section{Results and Discussion}

\section{1. ph Values to Determine Food Freshness}

Changes in $\mathrm{pH}$ values on the surface of the beef and fish samples are useful in tracking the freshness of food samples [17-19]. The accumulation of lactic acid after post mortem glycolysis causes a decrease in $\mathrm{pH}$. After this initial phase, $\mathrm{pH}$ increases as volatile basic amines are produced from the 
spoilage of food [20,21]. Thus, to determine time points of food spoilage, we measured $\mathrm{pH}$ on food samples as an indicator of freshness.

In beef, $\mathrm{pH}$ ranging from 5.7 to 6.0 is considered to be fresh, while meat with a $\mathrm{pH}$ higher than 6.2 refers to dark, firm, and dry (DFD) meat, which is susceptible to the growth of microorganisms [22,23]. As shown in Figure 2a, the $\mathrm{pH}$ of our beef sample increased from 5.9 to 6.5 during the 30-h period of measurement. We identified beef in the "fresh" state during the initial 2-h period when its $\mathrm{pH}$ was lower than 6.0. After a 23-h incubation, the $\mathrm{pH}$ became higher than 6.2, and we categorized the beef's state as "spoiled". We termed beef as "likely spoiled" between 2 and $23 \mathrm{~h}$ when $\mathrm{pH}$ values were 6.0-6.2.

In fresh tuna, $\mathrm{pH}$ is between 5.2 and 6.1, while $\mathrm{pH}$ is between 6.1 and 6.3 in fresh salmon [24-26]. Unlike beef, fish is frozen immediately after catching, which is why we observed decreases in $\mathrm{pH}$ in most of our fish samples during initial measurements. In tuna, $\mathrm{pH}$ decreased from 6.1 to 5.9 during the first 7-h measurement. Then, the $\mathrm{pH}$ value increased to 6.0 for $4 \mathrm{~h}$ and remained at $\mathrm{pH} 6.0$ until the end of the measurement (Figure $2 b$ ).

In Atlantic salmon, the $\mathrm{pH}$ decreased from 6.3 to 6.1 during the first $9 \mathrm{~h}$ of the experiment. Then, the $\mathrm{pH}$ value increased rapidly for $4 \mathrm{~h}$ until it reached $\mathrm{pH}$ 6.2. For the next $17 \mathrm{~h}$, the $\mathrm{pH}$ slowly increased and plateaued at 6.4 (Figure 2c). Since Pacific salmon (Oncorhynchus nerka) is known to spoil faster than Atlantic salmon [27], we observed decreases in $\mathrm{pH}$ only for the first $2 \mathrm{~h}$ of the experiment. Then, the $\mathrm{pH}$ increased rapidly for $10 \mathrm{~h}$ until it reached $\mathrm{pH} 6.5$ and remained the same for $18 \mathrm{~h}$ (Figure 2d). Although the absolute $\mathrm{pH}$ values were different, $\mathrm{pH}$ changes in tuna seemed to be similar with those of Atlantic salmon. Based on $\mathrm{pH}$ measurements, in fish, we define "fresh" for the initial $2 \mathrm{~h}$ when the $\mathrm{pH}$ started decreasing and "spoiled" after 12 to $13 \mathrm{~h}$ when the $\mathrm{pH}$ value plateaued at $\mathrm{pH}$ 6.4-6.5 for salmon and $\mathrm{pH} 6.0$ for tuna, respectively. We termed "likely spoiled" between the time periods of "fresh" and "spoiled" when the $\mathrm{pH}$ values changed dramatically.
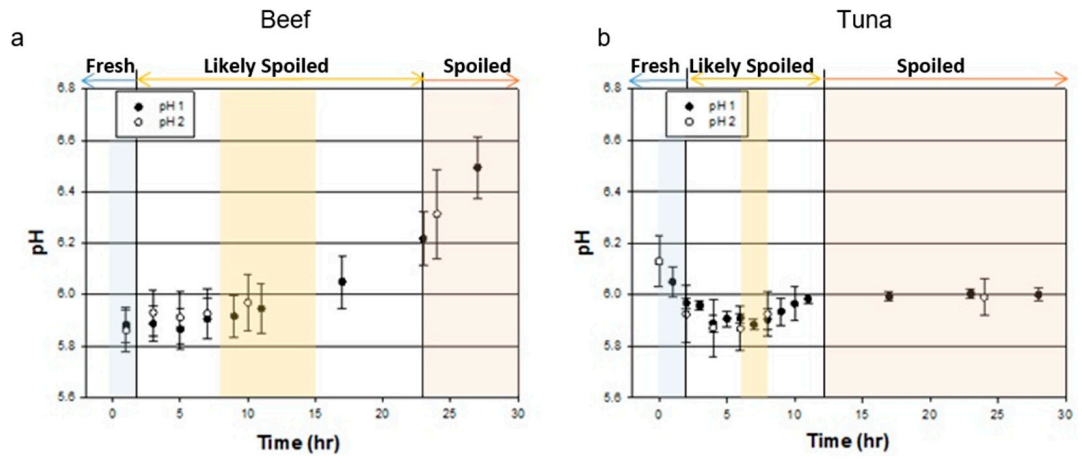

C

Salmon (Atlantic)

d

Salmon (Pacific)
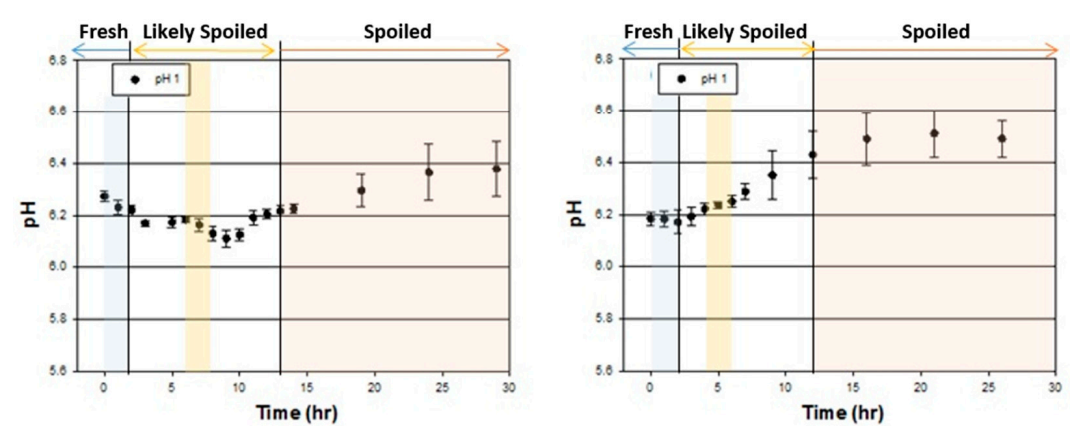

Figure 2. Time series of $\mathrm{pH}$ values for food samples to determine freshness: (a) beef, (b) tuna, (c) Atlantic salmon, and (d) Pacific salmon. At each time slot, the data point is for the mean of 60 measurements (3 sample $\times 20$ measurements), and the bar represents the standard deviation of them. There were two separate rotting processes in the beef and tuna experiments, which are presented as $\mathrm{pH} 1$ and $\mathrm{pH} 2$. Spectral response data collected during the shaded time slots (blue, yellow, and red) were used for the machine learning model. 


\subsection{Staphylococcus Aureus and TBARS}

The growth of Staphylococcus Aureus (S. Aureus), which is responsible for food poisoning through enterotoxin production, is frequently used as a biological indicator of food freshness [28].

To support our $\mathrm{pH}$ data, we cultured S. Aureus from beef and fish samples at each time point representing states of food freshness. S. Aureus is Gram-positive bacteria causing food poisoning. Since it survives well in the harsh environment through the formation of biofilm, the growth of S. Aureus is found in a wide range of food [29]. After a $24-\mathrm{h}$ incubation at $37{ }^{\circ} \mathrm{C}$, the number of S. Aureus colonies on Baird Parker (BP) selective media plates (Millipore Sigma) was counted (Figure 3). In all four samples, we found that $S$. Aureus colonies were significantly increased at the time when food was "spoiled". We also showed that significant changes in colony numbers when samples were "likely spoiled", indicating microbiological changes in food.
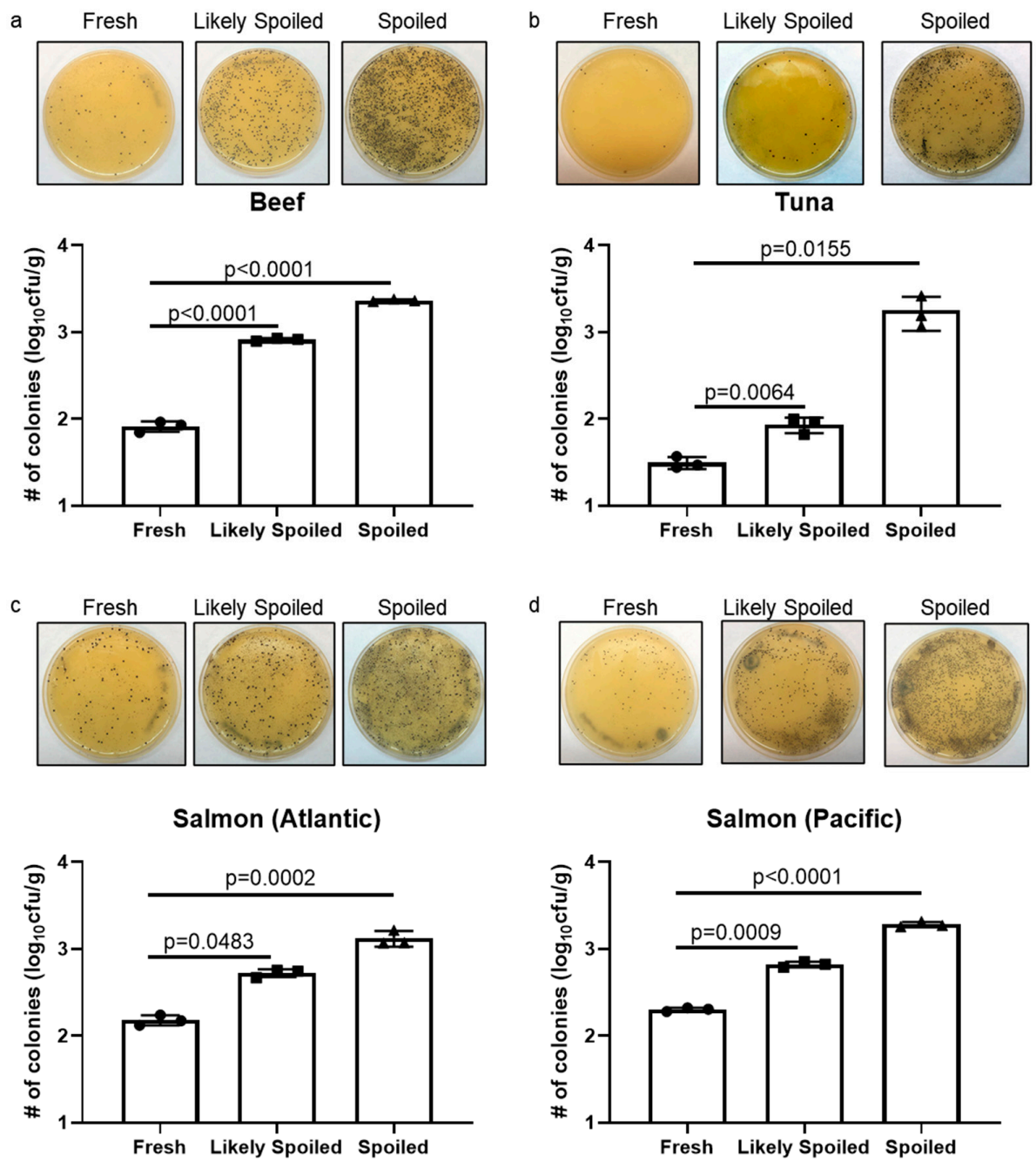

Figure 3. Growth of Streptococcus Aureus as an indicator of food freshness.

To further determine food freshness using a biochemical method, lipid oxidation due to the degradation of fat from food samples was measured using thiobarbituric acid reactive substances (TBARS) assay [30]. Except for Pacific salmon, levels of malondialdehyde (MDA), a by-product of TBA activity, were significantly higher in the spoiled food samples (Figure 4). Although they did not meet statistical significance, there was a tendency of increases in MDA levels at the time point when food was "likely spoiled". 

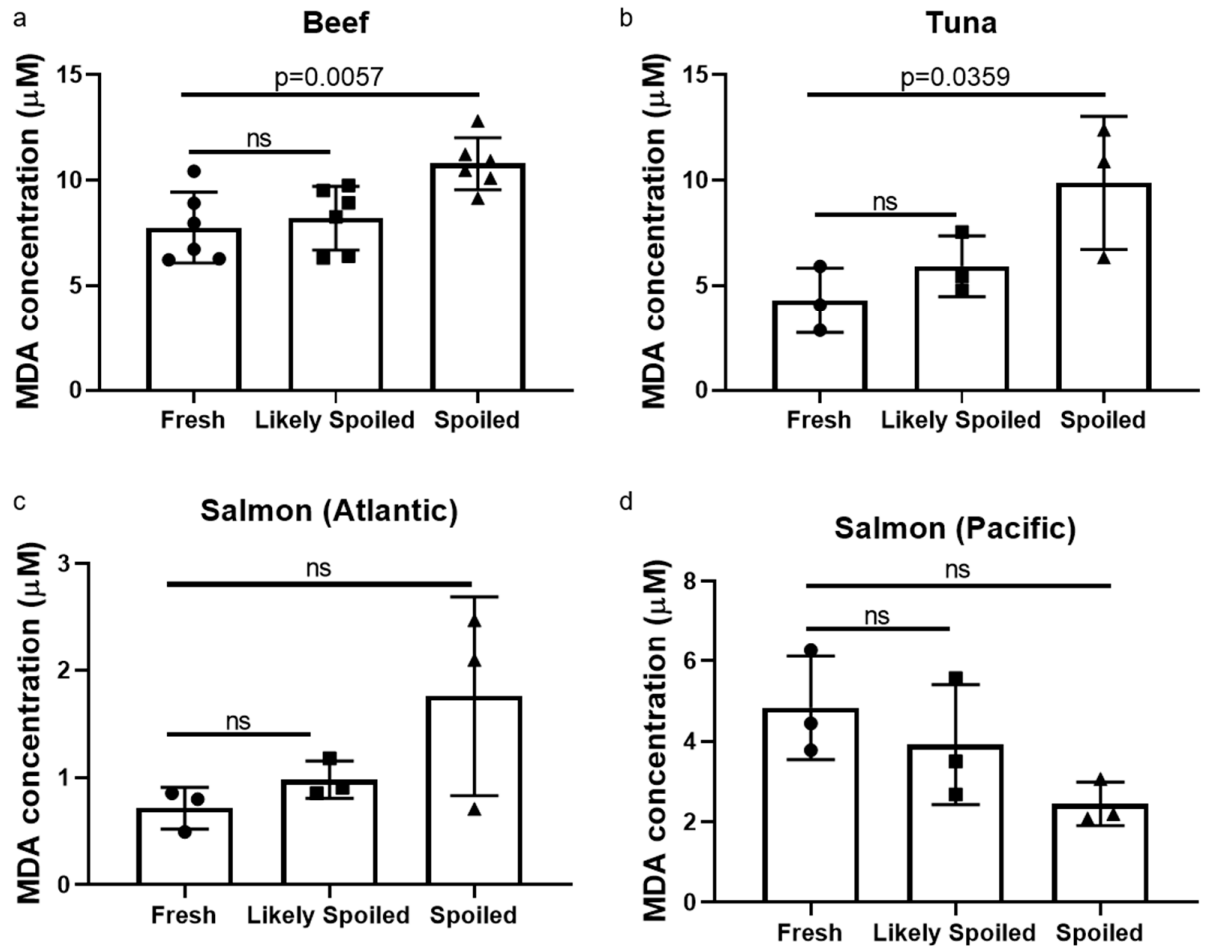

Figure 4. Thiobarbituric acid reacted substance (TBARS) assay to evaluate food freshness (ns: not significant).

\subsection{Data Classification}

Spectral response data were automatically taken every minute using LinkSquare ${ }^{\circledR}$ with two light sources: an LED and a bulb. These two light sources produced two spectrum data peaking around $600 \mathrm{~nm}$ (LED) and $900 \mathrm{~nm}$ (bulb). Since data were collected continuously, over 1000 graphs were averaged to show one spectral response data graph for each status. To minimize statistical errors, we kept the same number (approximately 1000 spectra per each category, as shown in Table 2) for each classification model by focusing on the dataset obtained from time points as color coded in Figure 5.

Table 2. The number of spectra collected for each food category.

\begin{tabular}{ccccc}
\hline & Salmon (Atlantic) & Salmon (Pacific) & Tuna & Beef \\
\hline Fresh & 1031 & 1055 & 840 & 1545 \\
\hline Likely spoiled & 1042 & 1095 & 978 & 1803 \\
\hline Spoiled & 1129 & 1457 & 1045 & 1694 \\
\hline
\end{tabular}

Meat pigment consists of heme proteins such as hemoglobin and cytochrome C. Deoxyhemoglobin specifically promotes the oxidization of fat and darkens the color [31]. As shown in Figure 5a(1-3), spectral responses between 550 and $650 \mathrm{~nm}$ were different between "fresh" and "spoiled" beef, indicating color changes resulting from the oxidization of heme proteins.

In both tuna and Atlantic salmon, we observed slight decreases in the intensity of bulb spectral response between 950 and $1000 \mathrm{~nm}$ (Figure 5(b4,c4)) when comparing "spoiled" samples to "fresh" ones. Since it is known that spectral responses in these wavelengths are highly influenced by the overtone band of water [32], the increased water content on the surface of "spoiled" samples seemed to drop the intensity of the spectral response.

In salmon, carotenoids, astaxanthin, and canthaxanthin are pigments that absorb light under a $600 \mathrm{~nm}$ wavelength. Since these spectrum ranges are overlapped with absorbing regions of heme 
protein, which determine the freshness of salmon ${ }^{33}$, it is more useful to evaluate differences between 605 and $735 \mathrm{~nm}$. Although changes were not explicit, there was a trend of increased intensities in both Atlantic and Pacific salmons when they were "spoiled" (Figure 5c,d).
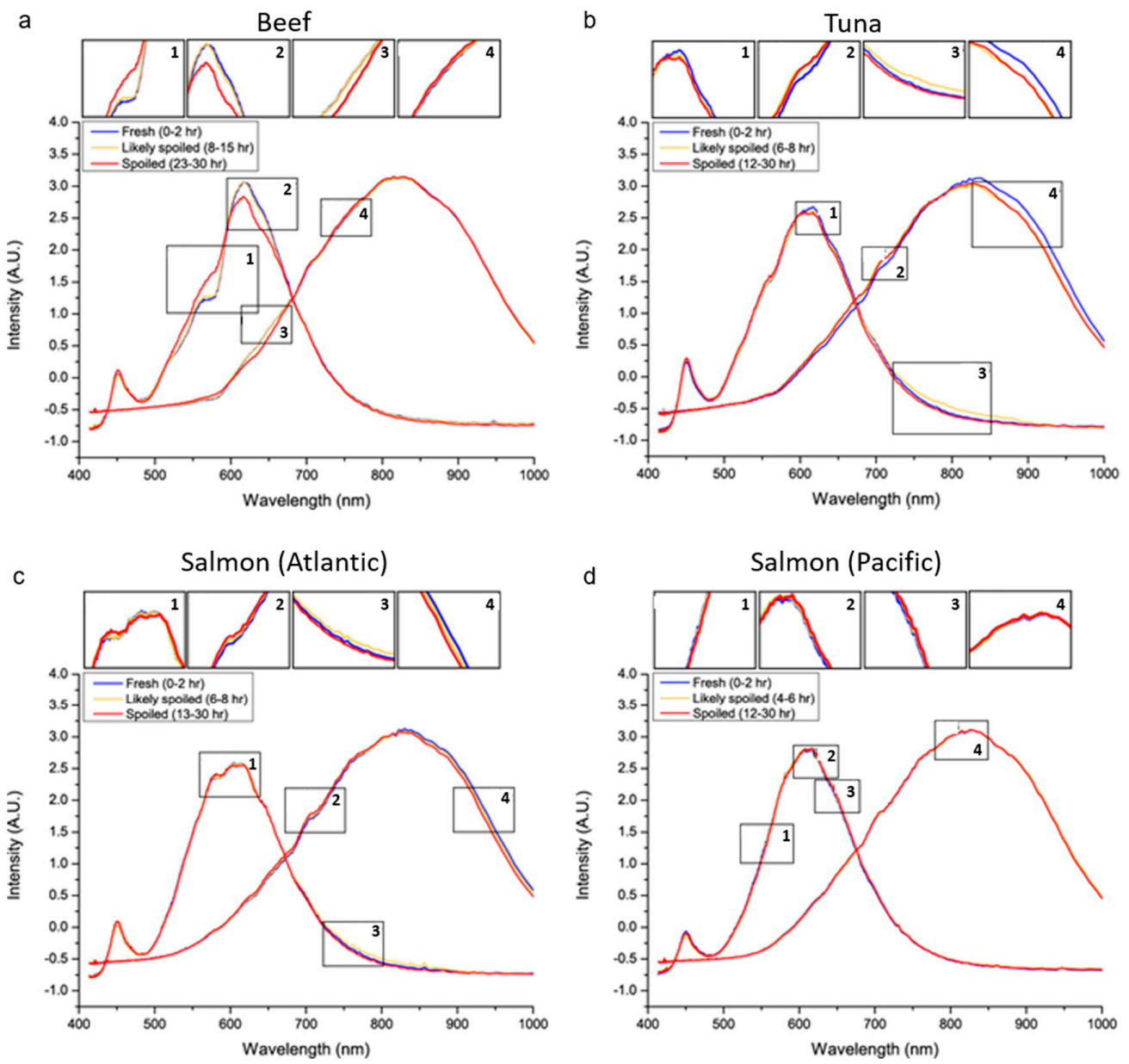

Figure 5. Spectral responses reflecting states of food freshness: (a) beef, (b) tuna, (c) Atlantic salmon, and (d) Pacific salmon. Average spectrum over 1000 spectra per each state after Standard Normal Variate (SNV) transformation are shown in the plots. Blue lines are for "Fresh", yellow lines are for "Likely Spoiled", and red lines for "Spoiled" samples. The lines with the maximum peak around $600 \mathrm{~nm}$ are the reflected spectrum of the LED light, and the lines with the peak around $800 \mathrm{~nm}$ are the reflected spectrum of the bulb light. For each plot, four areas with significant differences between the graphs are enlarged above for illustration purposes.

Whether differences were prominent or not, these spectrum changes among "fresh", "likely spoiled", and "spoiled" were taken into consideration when applying to our machine learning algorithm for classification.

\subsection{Validation}

To analyze and classify the collected spectrum data, we divided them into two groups: the first group for training and the second group for verification. Data in both groups were collected independently to ensure the validity of the verification process. The first group was used for training to build a CNN-based classification model. To evaluate the accuracy of the model, we ran the obtained classification model with the second set for verification. The obtained confusion matrix is shown in Table 3. Rows in the table indicate the number of the spectral response from the known categories, and columns show three possible classification results that our model led to. 
Thus, the diagonal values of the confusion matrix represent the true positives of each class, while the others determine errors. The total accuracy can be calculated through the sum of diagonal values over all values. The total accuracy was $85 \%$ for salmon ( $84 \%$ for Atlantic salmon and $85 \%$ for Pacific salmon), $88 \%$ for tuna, and $92 \%$ for beef, indicating that the handheld VIS/NIR spectrometer with our CNN-based classification model can assess the freshness of food with high accuracy [33].

Table 3. Confusion matrices of the machine learning models using the independently collected verification set.

\begin{tabular}{|c|c|c|c|c|}
\hline \multicolumn{5}{|c|}{ (a) Salmon (Atlantic) } \\
\hline & Fresh & Likely Spoiled & Spoiled & Accuracy \\
\hline Fresh & 164 & 26 & 7 & $83 \%$ \\
\hline Likely spoiled & 23 & 157 & 14 & $81 \%$ \\
\hline Spoiled & 14 & 32 & 319 & $87 \%$ \\
\hline \multicolumn{5}{|c|}{ (b) Salmon (Pacific) } \\
\hline & Fresh & Likely Spoiled & Spoiled & Accuracy \\
\hline Fresh & 73 & 9 & 4 & $85 \%$ \\
\hline Likely spoiled & 10 & 68 & 12 & $76 \%$ \\
\hline Spoiled & 3 & 7 & 136 & $93 \%$ \\
\hline
\end{tabular}

(c) Tuna

\begin{tabular}{ccccc}
\hline & Fresh & Likely Spoiled & Spoiled & Accuracy \\
\hline Fresh & 153 & 4 & 9 & $92 \%$ \\
\hline Likely spoiled & 23 & 168 & 12 & $83 \%$ \\
\hline Spoiled & 8 & 29 & 293 & $89 \%$ \\
\hline
\end{tabular}

(d) Beef

\begin{tabular}{ccccc}
\hline & Fresh & Likely Spoiled & Spoiled & Accuracy \\
\hline Fresh & 134 & 18 & 3 & $86 \%$ \\
\hline Likely spoiled & 20 & 259 & 2 & $92 \%$ \\
\hline Spoiled & 3 & 9 & 258 & $96 \%$ \\
\hline
\end{tabular}

The validation of our CNN-based machine learning algorithm can be more accurately explained by its sensitivity and specificity. Since our CNN-based classification model has three possible predictions, "fresh", "likely spoiled", and "spoiled", it is necessary to change the current three-class classification test into three binary classification tests. For example, Figure 6a shows the confusion matrix of Atlantic salmon, which is the same as that in Table 1 The following Figure $4 \mathrm{~b}$ is a modified two-by-two $(2 \times 2)$ confusion matrix assuming the binary classification asking if a spectral response of a sample is "fresh". True Positive (TP) indicates the cases in which the actual "fresh" spectral responses are predicted correctly as "fresh". False Positive (FP) indicates the cases in which the actual "not fresh" spectral responses are predicted incorrectly as "fresh". False Negative (FN) indicates the cases in which the actual "fresh" spectral responses are predicted incorrectly as "not fresh". True Negative (TN) indicates the cases in which the actual "not fresh" spectral responses are predicted correctly as "not fresh." The sensitivity of the Atlantic salmon is 0.83 for "fresh" binary classification test, based on Equation (1). The specificity can be calculated similarly. When the sensitivity and specificity values are calculated for all three binary classification tests, Table 4 is obtained. It shows relatively high numbers, which also validates the possibility of using our $\mathrm{CNN}$-based classification model. 
a

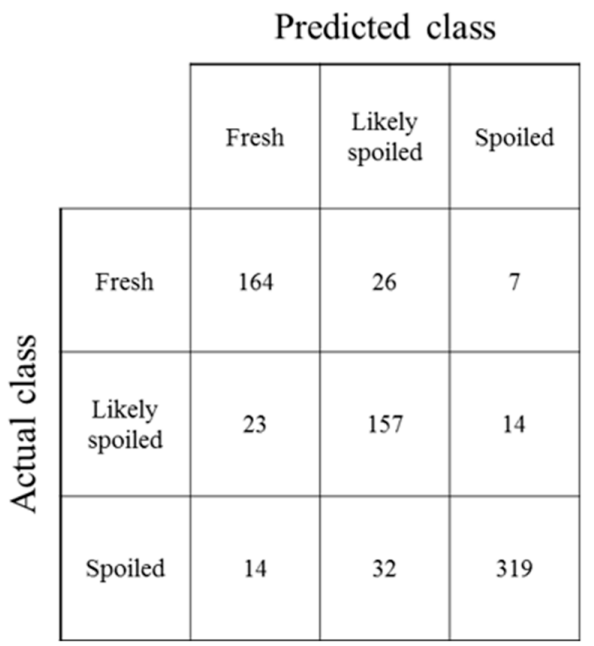

b

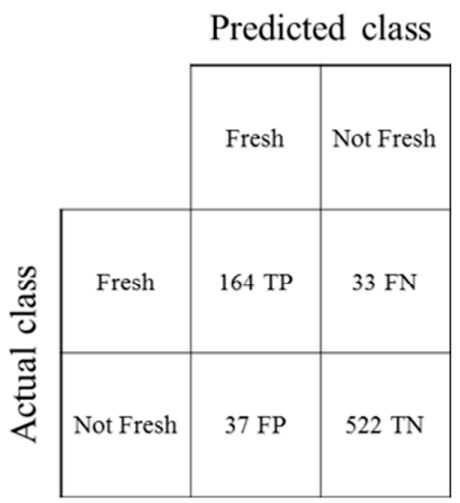

Figure 6. Confusion matrix (a) for a conventional two-by-two $(2 \times 2)$ matrix $(\mathbf{b})$ of Salmon (Atlantic) "fresh": TP is the True Positive of "fresh", FP is the False Positive of "fresh", FN is the False Negative of "fresh", and TN is the True Negative of "fresh".

Table 4. Calculation of sensitivity and specificity for four machine learning models. (a) Sensitivities show how well the models give correct answers for each class; (b) Specificities show how well the models reject the spectral response data from other classes. Both sensitivity and specificity are high enough to demonstrate the performance of machine learning models.

\begin{tabular}{|c|c|c|c|c|}
\hline \multicolumn{5}{|c|}{ (a) Sensitivity } \\
\hline & Salmon (Atlantic) & Salmon (Pacific) & Tuna & Beef \\
\hline Fresh & 0.83 & 0.85 & 0.92 & 0.86 \\
\hline Likely spoiled & 0.81 & 0.76 & 0.83 & 0.92 \\
\hline Spoiled & 0.87 & 0.93 & 0.89 & 0.96 \\
\hline \multicolumn{5}{|c|}{ (b) Specificity } \\
\hline & Salmon (Atlantic) & Salmon (Pacific) & Tuna & Beef \\
\hline Fresh & 0.93 & 0.94 & 0.94 & 0.96 \\
\hline Likely spoiled & 0.90 & 0.93 & 0.93 & 0.94 \\
\hline Spoiled & 0.95 & 0.91 & 0.94 & 0.99 \\
\hline
\end{tabular}

\section{Conclusions}

We have developed a methodological approach to utilize a portable VIS/NIR spectrometer to assess the freshness of four food categories. The accuracy was $85 \%$ for salmon, $88 \%$ for tuna, and $92 \%$ for beef. Using the spectral response from a VIS-NIR portable spectrometer and a CNN-based machine learning-assisted classification, we have demonstrated a non-destructive, real-time measurement of food freshness. In addition, a CNN-based machine learning algorithm with a shift-invariant feature can minimize the effect of the variation caused using multiple devices in a real environment. We believe that this method can be easily applied to other foods and/or drinks, which will revolutionize the qualitative freshness measurement for consumers.

Author Contributions: Y.K., Y.N., and J.H.L. designed the study and interpreted the results. E.J.M., Y.K., Y.X., Y.N., and J.H.L. collected test data and drafted the manuscript. E.J.M. and A.J.G. contributed to the interpretation of the results. E.J.M. took the lead in writing the manuscript. All authors have read and agreed to the published version of the manuscript.

Funding: This work was partially supported by Institute for Information \& Communications Technology Promotion (IITP) grant funded by the Korean government (MSIP) (No. B0717-16-0065, Germanium on Silicon 
based Sensor which covers visible spectrum to Short Wavelength Infra-Red range (400-1600 nm) for Materials Identification and Application System Development) and partially supported by Korea Institute of Planning and Evaluation for Technology in Food, Agriculture, and Forestry (IPET) grant funded by the Korean government (Ministry of Agriculture and Forestry).

Acknowledgments: We would like to thank Matt Bogyo and Sumin Lee at Stanford University for their generosity to let us use their bacteria culture equipments for S. Aureus culture.

Conflicts of Interest: The authors declare no conflict of interest.

\section{References}

1. Lambert, A.D.; Smith, J.P.; Dodds, K.L. Shelf-Life Extension and Microbiological Safety of Fresh Meat-A Review. Food Microbiol. 1991, 8, 267-297. [CrossRef]

2. Olafsdottir, G.; Martinsdottir, E.; Oehlenschlager, J.; Dalgaard, P.; Jensen, B.; Undeland, I.; Mackie, I.M.; Henehan, G.; Nielsen, J.; Nilsen, H. Methods to evaluate fish freshness in research and industry. Trends Food Sci. Technol. 1997, 8, 258-265. [CrossRef]

3. Hasanah, U.; Setyowati, M.; Efendi, R.; Muslem, M.; Md Sani, N.D.; Safitri, E.; Yook Heng, L.; Idroes, R. Preparation and Characterization of a Pectin Membrane-Based Optical pH Sensor for Fish Freshness Monitoring. Biosensors (Basel) 2019, 9, 60. [CrossRef] [PubMed]

4. Brown, M.R.; Kube, P.D.; Taylor, R.S.; Elliott, N.G. Rapid compositional analysis of Atlantic salmon (Salmo salar) using visible-near infrared reflectance spectroscopy. Aquac. Res. 2014, 45, 798-811. [CrossRef]

5. Crichton, S.O.J.; Kirchner, S.M.; Porley, V.; Retz, S.; Von Gersdoiff, G.; Hensel, O.; Weygandt, M.; Sturm, B. Classification of organic beef freshness using VNIR hyperspectral imaging. Meat Sci. 2017, 129, $20-27$. [CrossRef]

6. Li, M.; Qian, Z.; East, A.R. Does consumer-scale near-infrared (NIR) spectroscopy provide opportunities for kiwifruit quality measurement. In ISHS Acta Horticulturae 1218: IX International Symposium on Kiwifruit; International Society for Horticultural Science (ISHS): Leuven, Belgium, 2018.

7. Lee, J.H.; Na, Y.; Kang, J. Spectrometers with Self- Compensation of Rotational Misalignment. U.S. Patent 10281327, 6 May 2019.

8. LINKSQUARE. Available online: https://trademarks.justia.com/868/68/linksquare-86868200.html (accessed on 18 July 2020).

9. Crocombe, R.A. Portable spectroscopy in 2019: Smaller, cheaper and in consumer products? In Proceedings of the SPIE 10983, Next-Generation Spectroscopic Technologies XII, Baltimore, MD, USA, 13 May 2019.

10. You, H.; Kim, Y.; Lee, J.H.; Jang, B.J.; Choi, S. Food Powder Classification Using a Portable Visible-Near-Infrared Spectrometer. J. Electromagn. Eng. Sci. 2017, 17, 186-190. [CrossRef]

11. Food Manufacture. Available online: https://www.foodmanufacture.co.uk/Article/2018/10/02/Food-safetyculture-identified-in-latest-global-standards (accessed on 18 July 2020).

12. Kanmani, R.; Sureshkumar, A.; Mugilan, A.; Praveena, V. Non-destructive Approach to Detect Pesticides in Fruits and Vegetables using IoT Technology. In Proceedings of the 2020 International Conference on Computer Communication and Informatics (ICCCI), Coimbatore, India, 22-24 January 2020.

13. LeCun, Y.; Huang, F.J.; Bottou, L. Learning methods for generic object recognition with invariance to pose and lighting. In Proceedings of the 2004 IEEE Computer Society Conference on Computer Vision and Pattern Recognition, Washington, DC, USA, 27 June-2 July 2004; Volume 2, pp. 11-104.

14. LeCun, Y.; Kavukcuoglu, K.; Farabet, C. Convolutional networks and applications in vision. In Proceedings of the 2010 IEEE international symposium on circuits and systems, Paris, France, 30 May-2 June 2010; pp. 253-256.

15. Abdalrahman, L.S.; Wells, H.; Fakhr, M.K. Staphylococcus aureus is More Prevalent in Retail Beef Livers than in Pork and other Beef Cuts. Pathogens 2015, 4, 182-198. [CrossRef]

16. Krizhevsky, A.; Sutskever, I.; Hinton, G.E. Imagenet classification with deep convolutional neural networks. In Proceedings of the Advances in Neural Information Processing Systems 25 (NIPS 2012), Lake Tahoe, CA, USA, 3 December 2012; pp. 1097-1105.

17. Gill, C.O. Meat Spoilage and Evaluation of the Potential Storage Life of Fresh Meat. J. Food Prot. 1983, 46, 444-452. [CrossRef] 
18. Heising, J.K.; Bartels, P.V.; Van Boekel, M.A.J.S.; Dekker, M. Non-destructive sensing of the freshness of packed cod fish using conductivity and pH electrodes. J. Food Eng. 2014, 124, 80-85. [CrossRef]

19. Huss, H.H. Quality and quality changes in fresh fish. In FAO Fisheries Technical Paper; Food and Agriculture Organization of the United Nations. Via delle Terme di Caracalla: Rome, Italy, 1995.

20. Kuswandi, B.; Nurfawaidi, A. On-package dual sensors label based on $\mathrm{pH}$ indicators for real-time monitoring of beef freshness. Food Control 2017, 82, 91-100. [CrossRef]

21. Miks-Krajnik, M.; Yoon, Y.J.; Ukuku, D.O.; Yuk, H.G. Volatile chemical spoilage indexes of raw Atlantic salmon (Salmo salar) stored under aerobic condition in relation to microbiological and sensory shelf lives. Food Microbiol. 2016, 53, 182-191. [CrossRef]

22. Newton, K.G.; Gill, C.O. The microbiology of DFD fresh meats: A review. Meat Sci. 1981, 5, $223-232$. [CrossRef]

23. Wieslaw Przybylski, D.H. Meat Quality: Genetic and Environmental Factors; Taylor \& Francis: Oxfordshire, UK, 2015; p. 488.

24. Abbas, K.A.; Ebrahimian, M. A review on correlation between fish freshness and $\mathrm{pH}$ during cold storage. Am. J. Biochem. Biotechnol. 2008, 4, 416-421. [CrossRef]

25. Celia, C.G.; Silva, D.J.B.D.P.; Maria, L.N. Enes Dapkeviciu, Storage temperature effect on histamine formation in big eye tuna and skipjack. J. Food Sci. 1998, 63, 644-647.

26. Eva Fathul Karamah, A.P.I. Nadifa Ismaningtyas, The applicaion of ozonated water to maintain the quality of tuna meat: The effect of contact time, contact temperature and ozone dosage. Iop Conf. Ser. Mater. Sci. Eng. 2019, 509, 1-10.

27. Erikson, U.; Beyer, A.R.; Sigholt, T. Muscle high-energy phosphates and stress affect K-values during ice storage of Atlantic salmon (Salmo salar). J. Food Sci. 1997, 62, 43-47. [CrossRef]

28. Schelin, J.; Wallin-Carlquist, N.; Cohn, M.T.; Lindqvist, R.; Barker, G.C.; Radstrom, P. The formation of Staphylococcus aureus enterotoxin in food environments and advances in risk assessment. Virulence 2011, 2, 580-592. [CrossRef]

29. Kadariya, J.; Smith, T.C.; Thapaliya, D. Staphylococcus aureus and staphylococcal food-borne disease: An ongoing challenge in public health. Biomed. Res. Int. 2014, 2014, 827965. [CrossRef]

30. Dominguez, R.; Pateiro, M.; Gagaoua, M.; Barba, F.J.; Zhang, W.; Lorenzo, J.M. A Comprehensive Review on Lipid Oxidation in Meat and Meat Products. Antioxidants (Basel) 2019, 8, 429. [CrossRef]

31. Mancini, R.A.; Hunt, M.C. Current research in meat color. Meat Sci. 2005, 71, 100-121. [CrossRef] [PubMed]

32. Nilsen, H.; Esaiassen, M.; Heia, K.; Sigernes, F. Visible/near-infrared spectroscopy: A new tool for the evaluation of fish freshness? J. Food Sci. 2002, 67, 1821-1826. [CrossRef]

33. Kimiya, T.; Sivertsen, A.H.; Heia, K. VIS/NIR spectroscopy for non-destructive freshness assessment of Atlantic salmon (Salmo salar L.) fillets. J. Food Eng. 2013, 116, 758-764. [CrossRef] 